\title{
Effects of Methamphetamine Toxicity on the Nervous System
}

\section{Sanaz Hadizade Asar*, Mohammad Hosseini-Sharifabad, Maryam Yadegari}

Departement of Anatomy and Cell Biology, Shahid Sadoughi University of Medical Sciences and Health Services, Yazd, Iran

\section{ABSTRACT}

Introduction: Methamphetamine (METH) is a drug which is highly addictive, psychoactive and hallucinogenic. Besides marijuana, it is the second widely-used drug all over the world, especially by young people and adolescents. The wide use of METH has opened a new era in social problems. It has been proved that these compounds have toxic effects on the central nervous system. Regular use of METH can damage the dopaminergic and serotonergic synaptic network in different parts of the brain. Memory and learning disorders, anxiety and depression as well as motor disorders, such as Parkinson's disease, are among the toxic side-effects of the drug on the neuronal system. In addition, evidence suggests that this compound can cause schizophrenia, cognitive disturbances, and motor disorders in some consumers. In spite of the devastating effects caused by METH, the exact mechanism of action has not been understood yet, which has created a major challenge to the treatment strategies. Conclusion: Today, using METH is highly increasing among the youth and teenagers. Sufficient knowledge and awareness on the catastrophic and irreversible side-effects of this drug can prevent serious harm to the public health.

\section{Key words:}

1. Methamphetamine

2. Central Nervous System

3. Peripheral Nervou System

*Corresponding Author: Sanaz Hadizade Asar

E-mail: sanaz.hadizade92@gmail.com 
اثرات سمّيت متامفتامين بر سيستم عصبى

ساناز هادى اده عصار"، محمد حسينى شريف آباد، مريم يادكارى

گروه علوم تشريح و بيولوزى، دانشكاه علوم يزشكى و خدمات بهداشتى -درمانى شهيد صدوقى، يزد، ايران

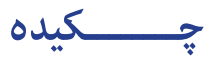

مقدمه: متامفتامين دارويى است كه بسيار اعتيادآور، توهمزاو سايكواكتيو است. در كنار مارىجوانا دومين

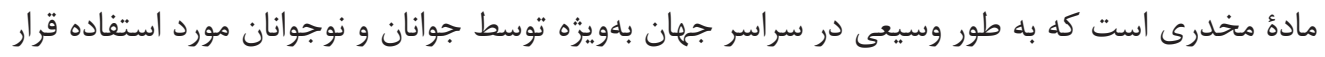

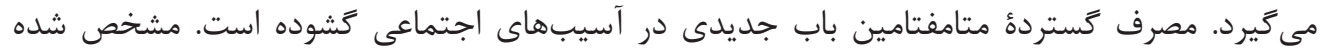

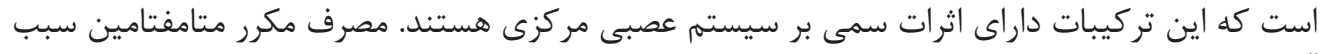

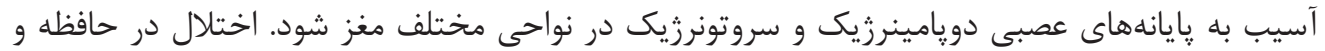

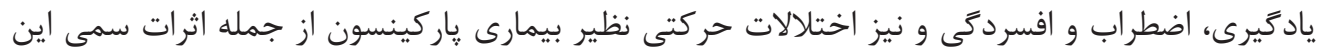

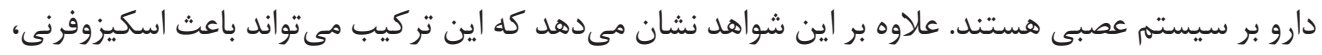

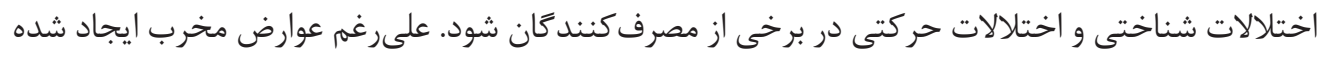

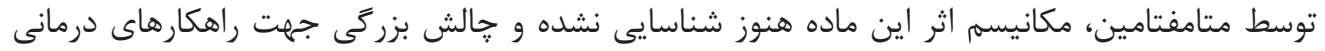

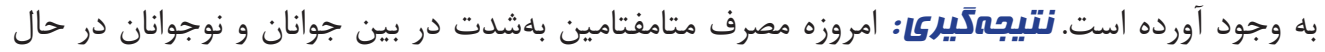

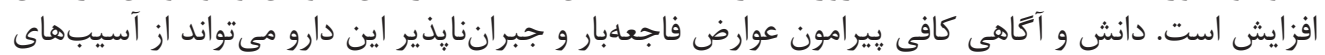

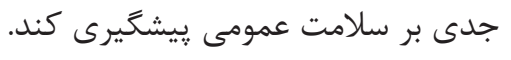

\footnotetext{
كليد وازمها:

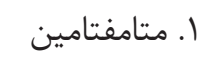

r. مركزى

محيطى سيستم عصبى
}

* نويسنده مسئول: ساناز هادىزاده عصار آدرس الكترونيكى: sanaz.hadizade92@gmail.com 


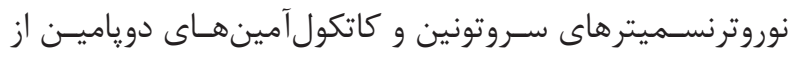

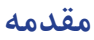

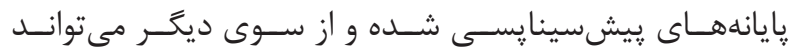

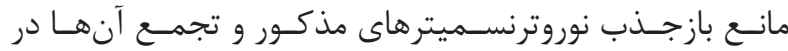

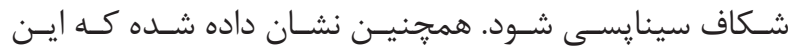

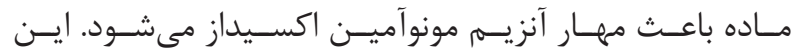

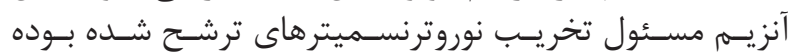

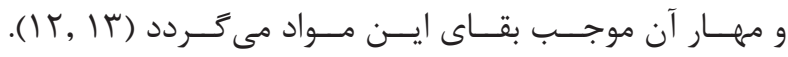

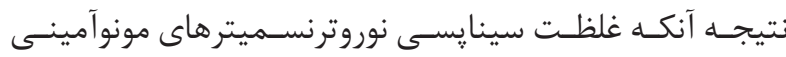

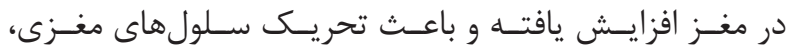

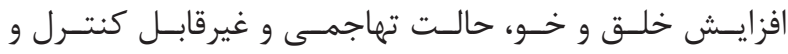

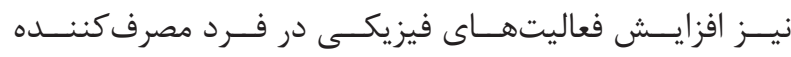

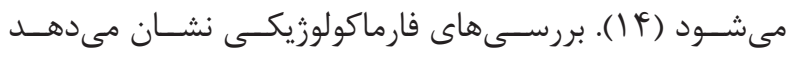

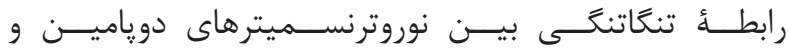

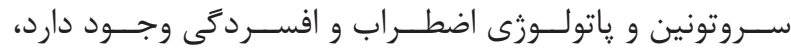

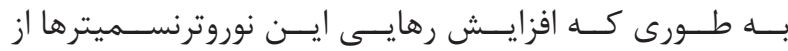

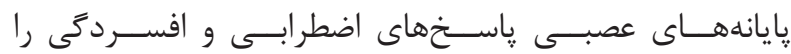

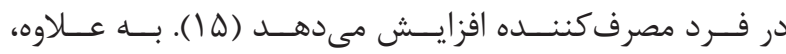

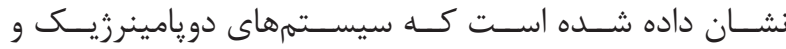

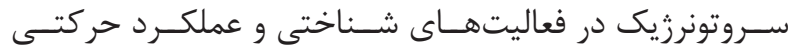

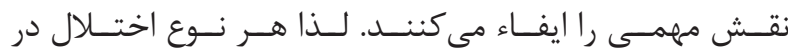

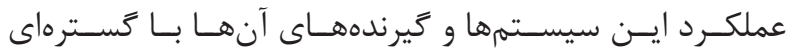

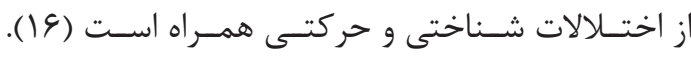

متامفتامين و نوروترنسميتر دويامين

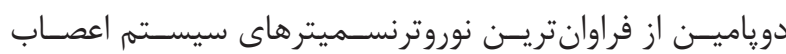

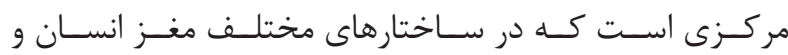

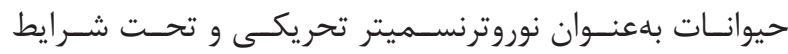

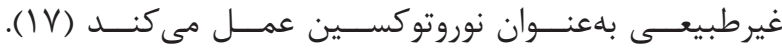

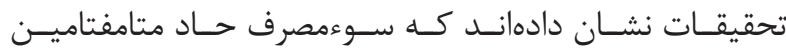

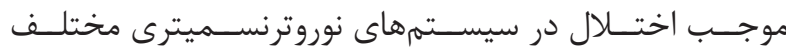

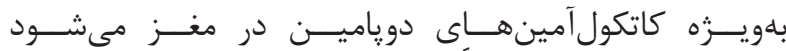

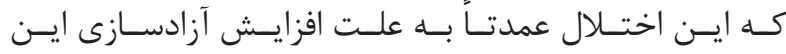

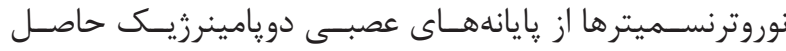

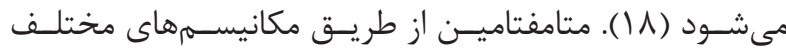

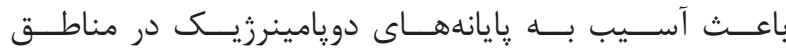

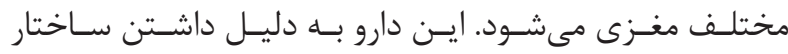

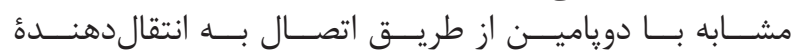

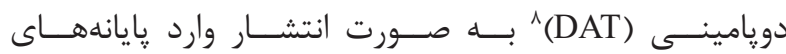

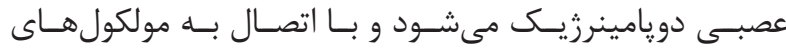

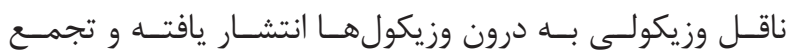

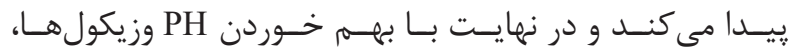

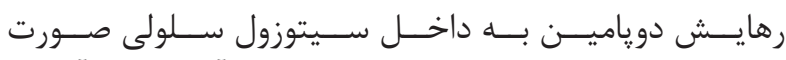

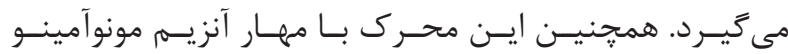

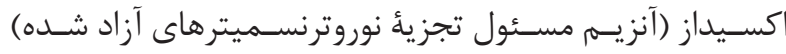

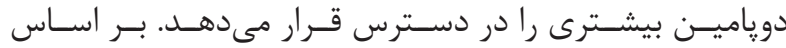

${ }^{1}$ Methamphetamine

${ }^{2}$ Crank

${ }^{3}$ Chalk

${ }^{4}$ Meth

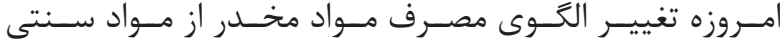

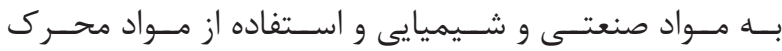

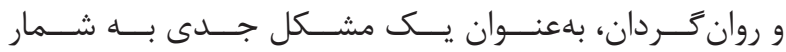

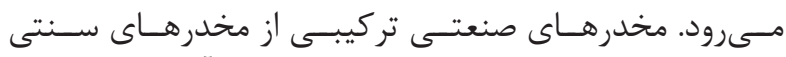

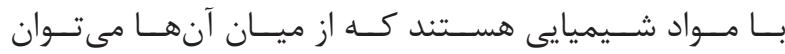

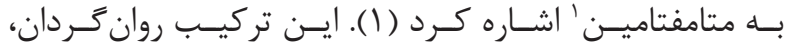

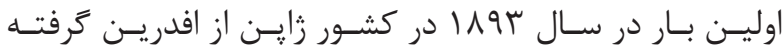

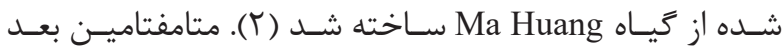

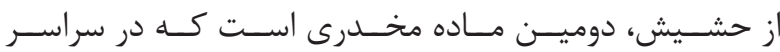

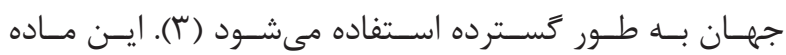

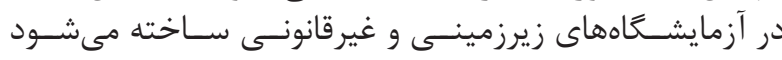

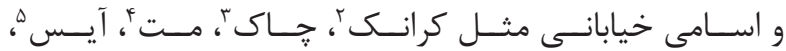

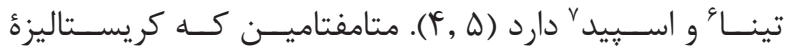

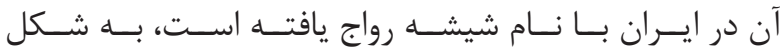

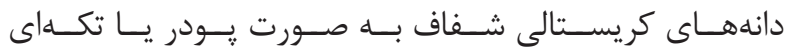

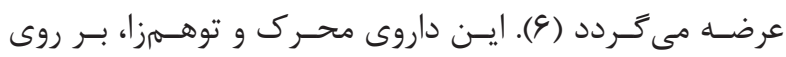

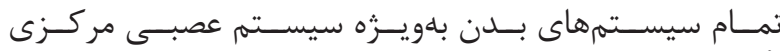

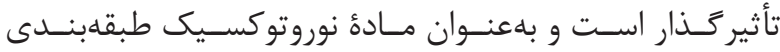

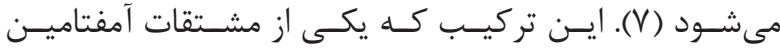

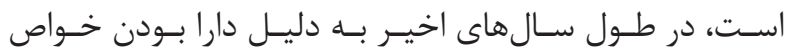

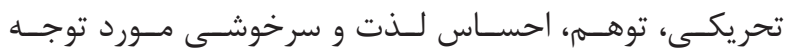

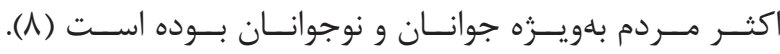

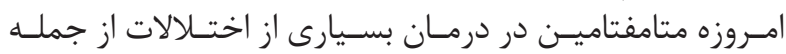

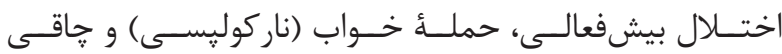

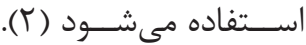

متامفتامين و اثرات نوروفار ماكولوزيكى مئى

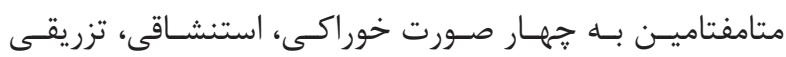

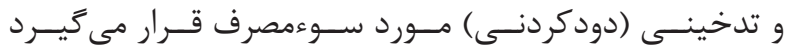

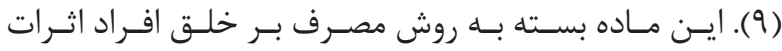

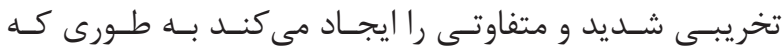

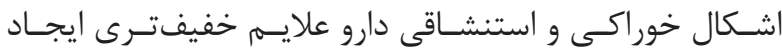

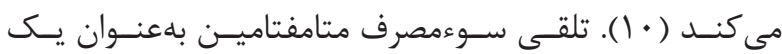

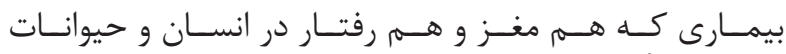

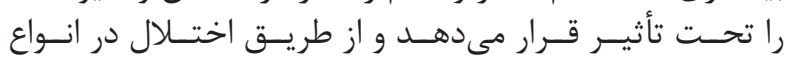

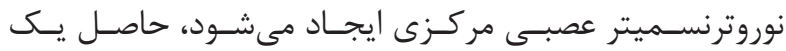

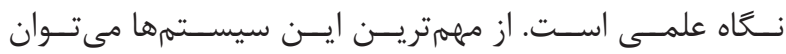

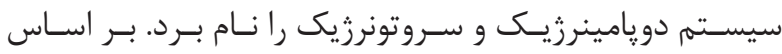

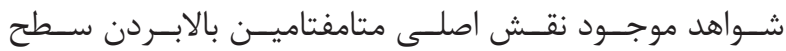

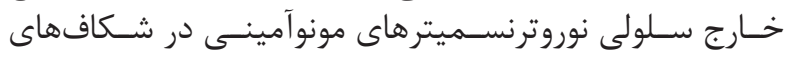

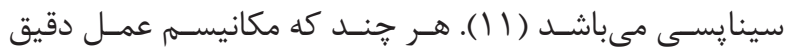

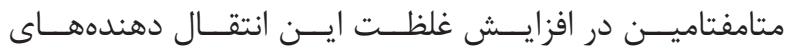

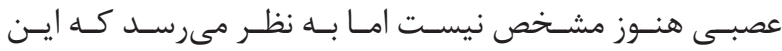

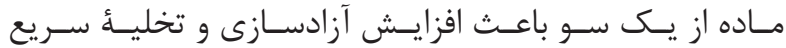

${ }^{5}$ Ice

${ }^{6}$ Tina

${ }^{7}$ Speed

${ }^{8}$ Dopamine transporter 


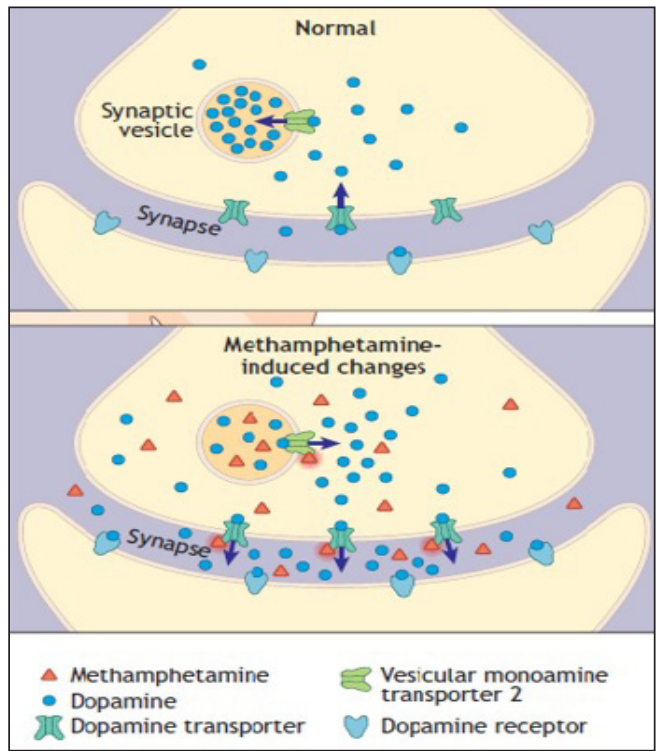

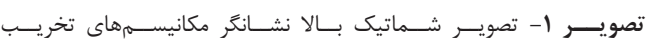

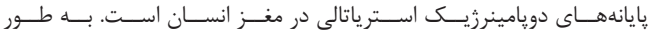

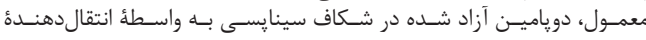

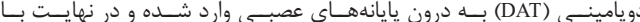

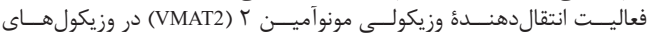

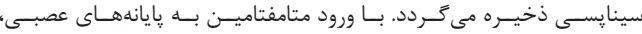

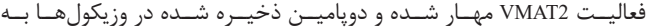

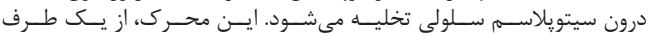

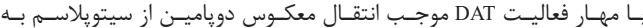

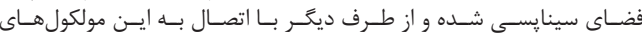

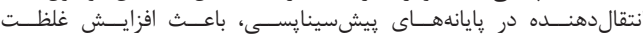

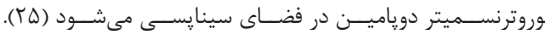

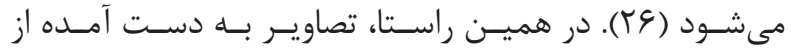

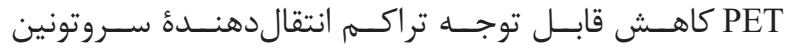

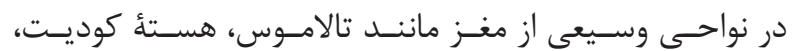

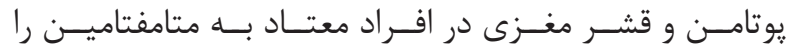

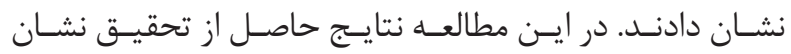

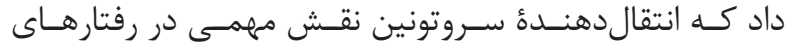

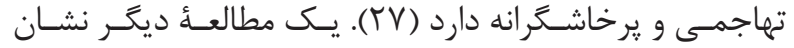

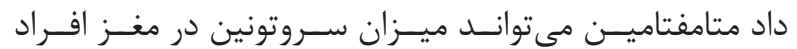

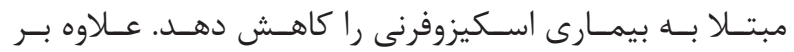

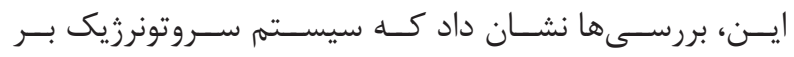

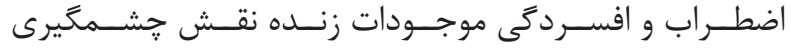

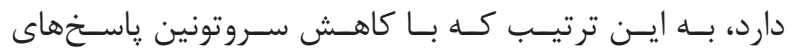

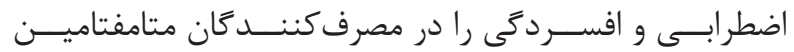

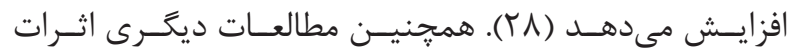

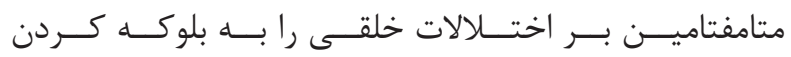

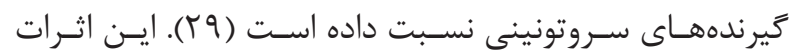

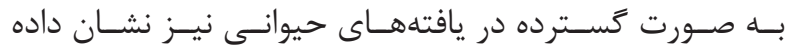

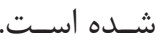

\section{متامفتامين و اختلالات شناختى}

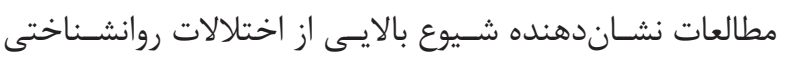

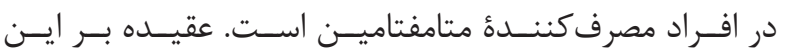

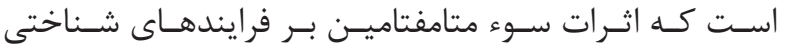

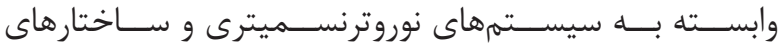

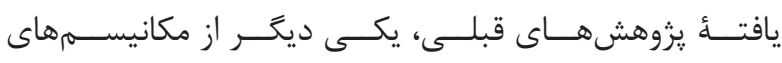

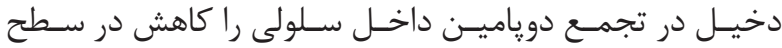

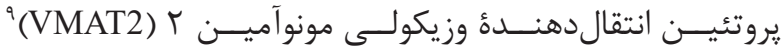

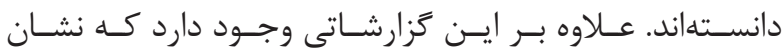

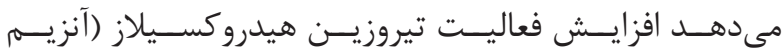

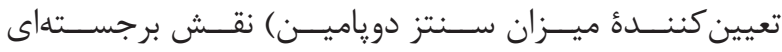

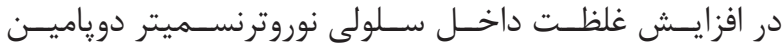

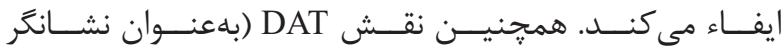

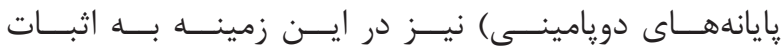

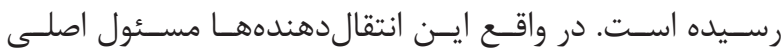

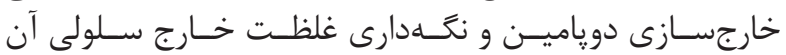

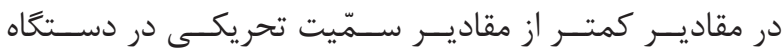

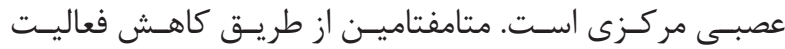

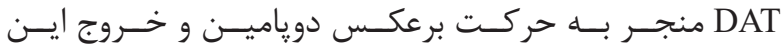

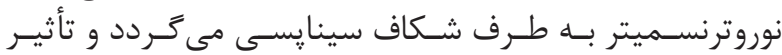

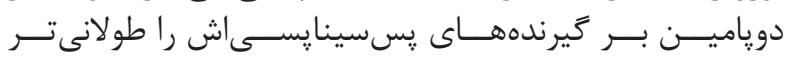

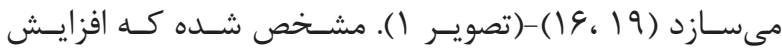

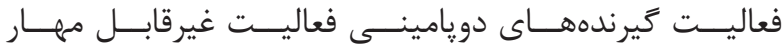

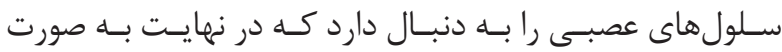

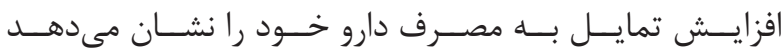

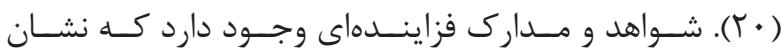

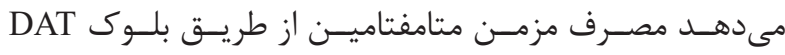

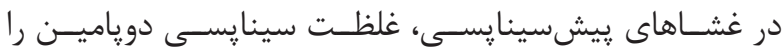

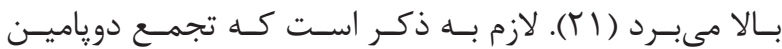

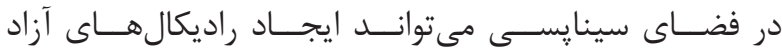

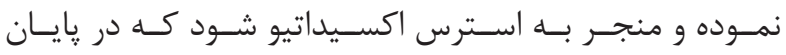

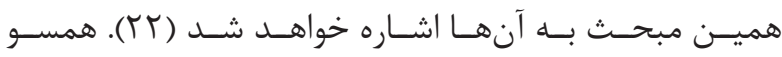

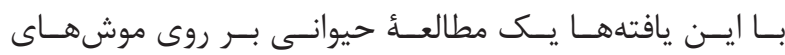

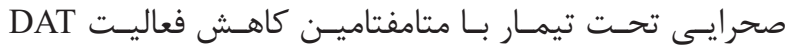

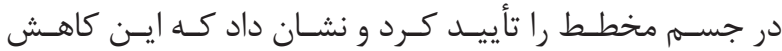

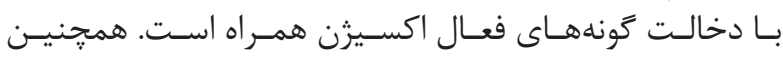

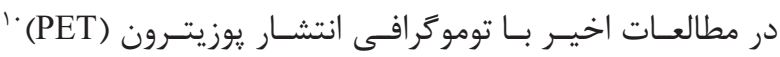

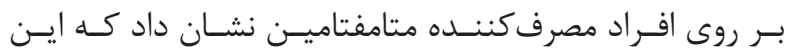

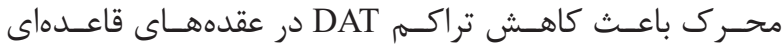

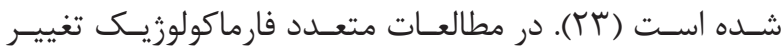

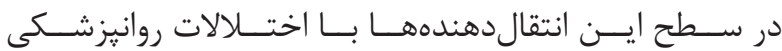

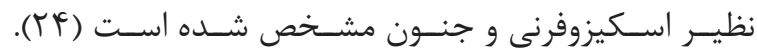
متامفتامين و نوروترنسميتر سروتونين

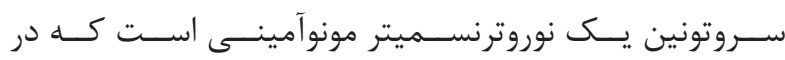

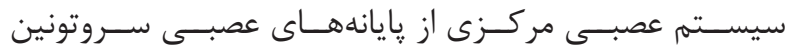

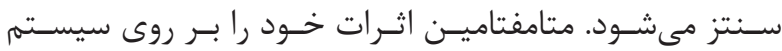

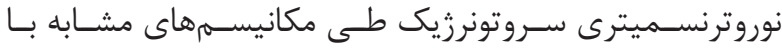

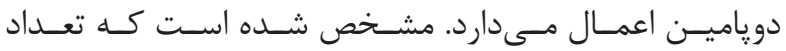

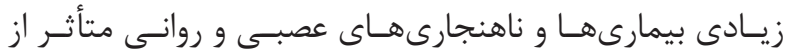

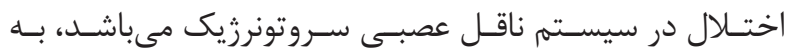

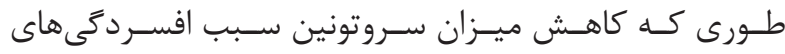

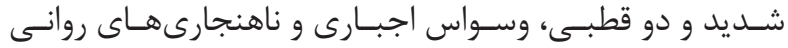

${ }^{9}$ Vesicular monoamine transporter 2

${ }^{10}$ Positron emission tomography 


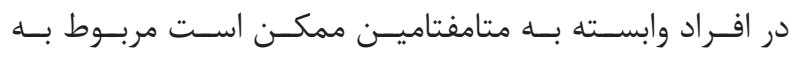

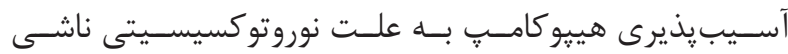

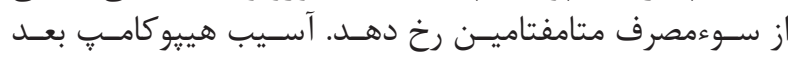

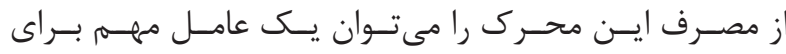

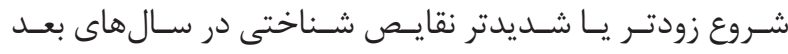

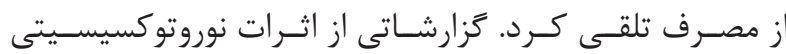

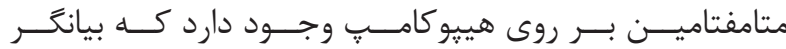

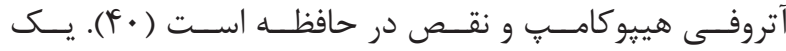

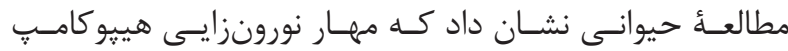

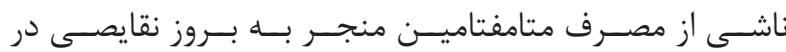

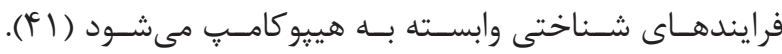

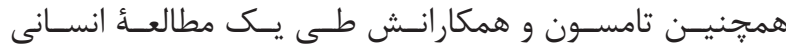

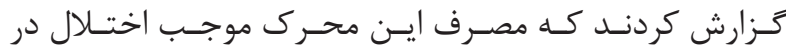

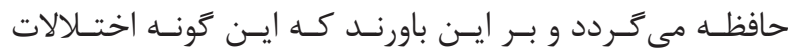

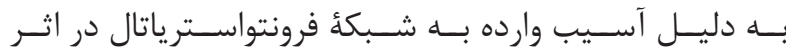

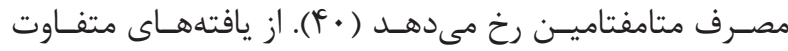

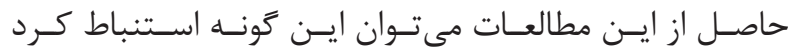

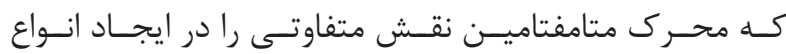

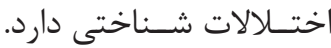
نوروتوكسيسيتى متامفتامين

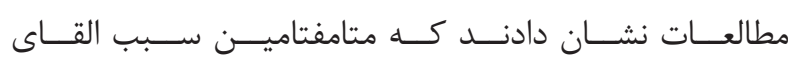

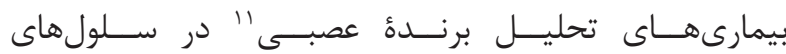

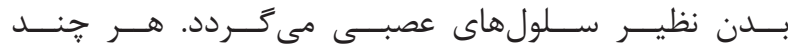

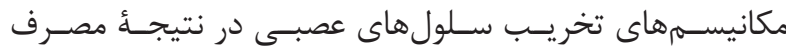

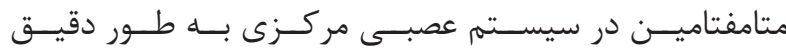

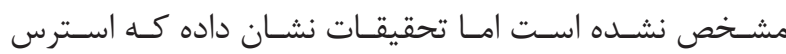

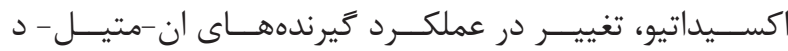

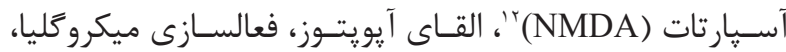

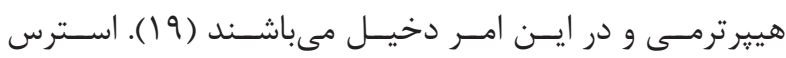

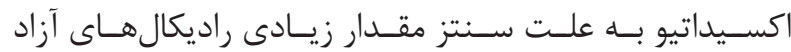

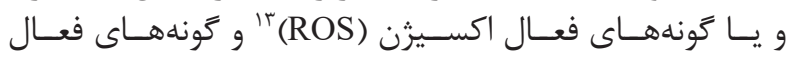

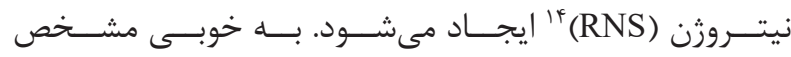

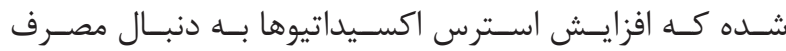

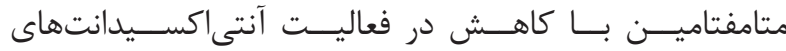

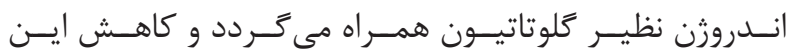

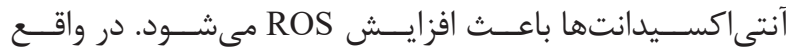

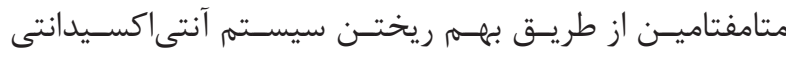

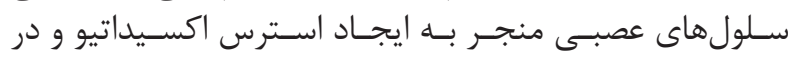

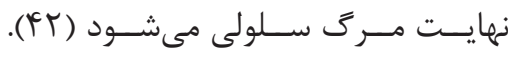

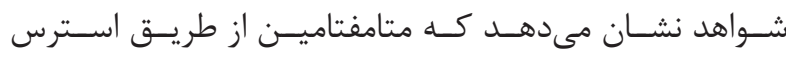

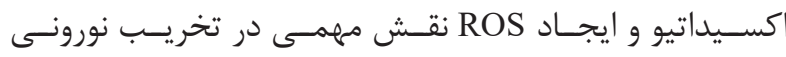

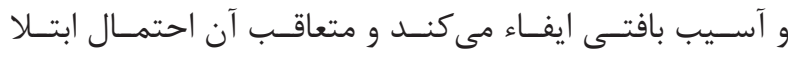

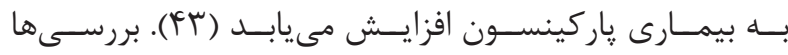

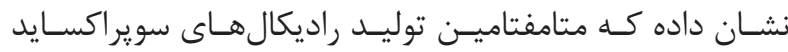

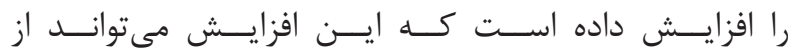

${ }^{11}$ Neurodegenerative

${ }^{12} \mathrm{~N}$-methyl-D-aspartate

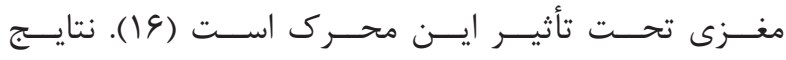

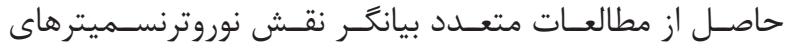

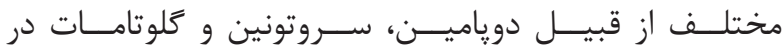

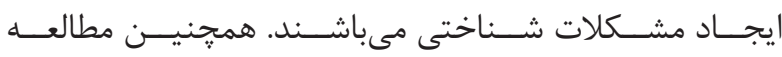

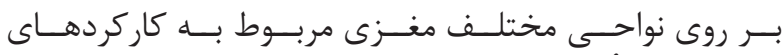

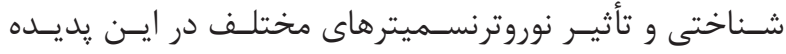

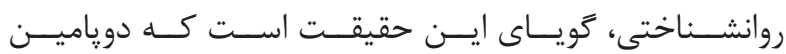

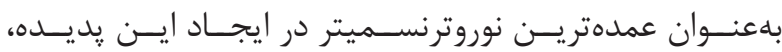

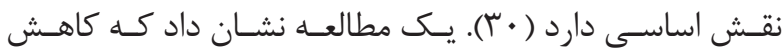

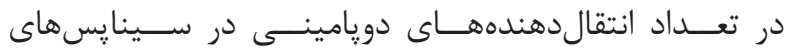

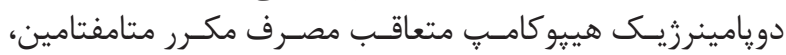

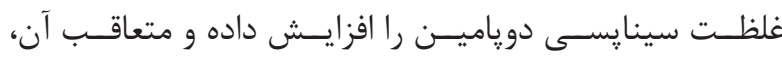

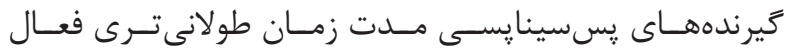

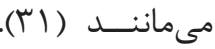

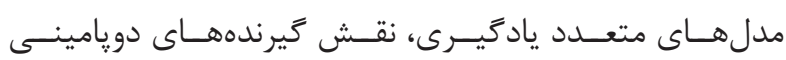

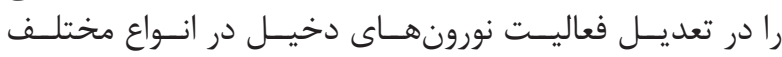

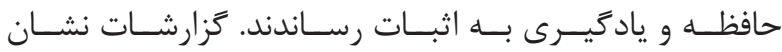

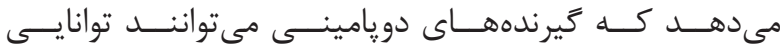

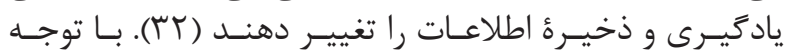

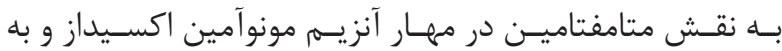

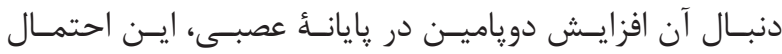

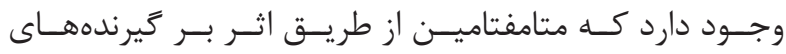

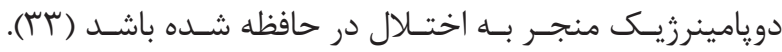

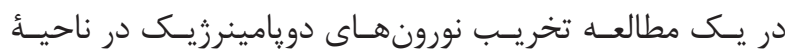

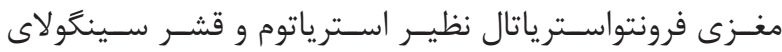

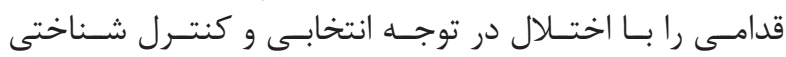

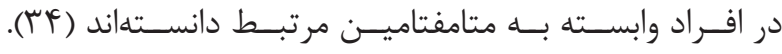

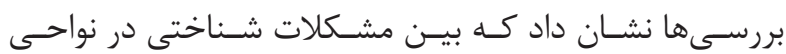

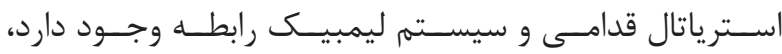

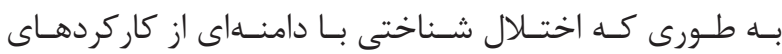

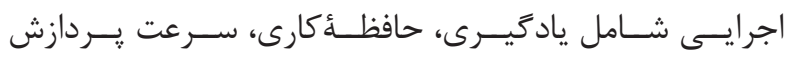

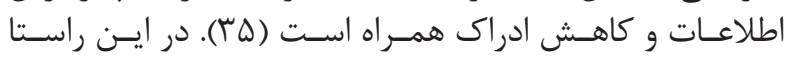

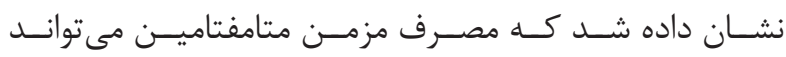

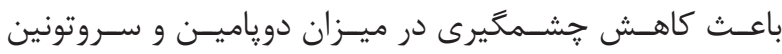

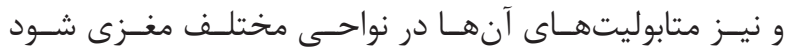

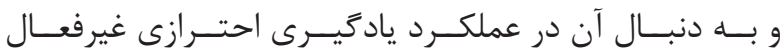

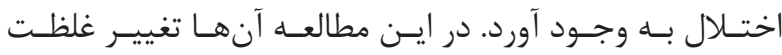

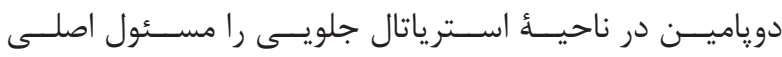

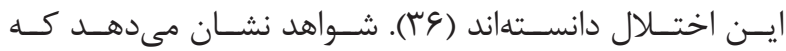

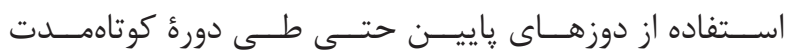

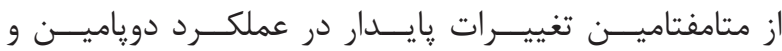

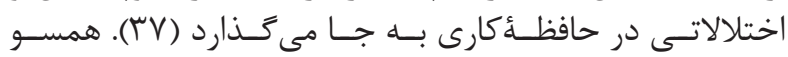

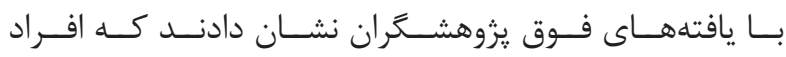

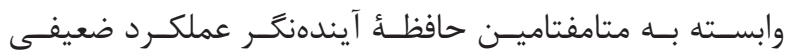

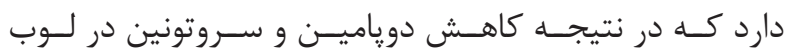

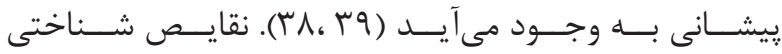

\footnotetext{
${ }^{13}$ Reactive oxygen species

${ }^{14}$ Reactive nitrogen species
} 


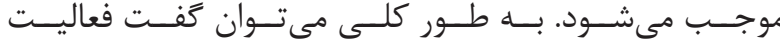

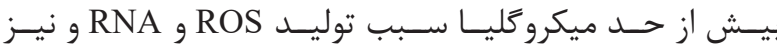

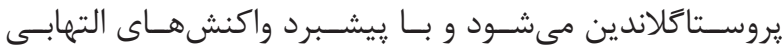

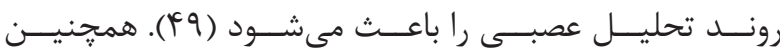

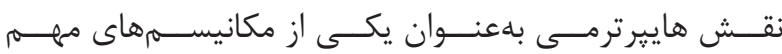

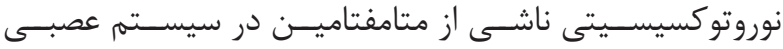

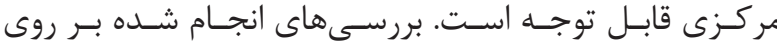

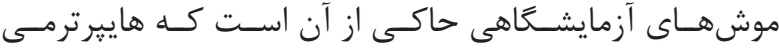

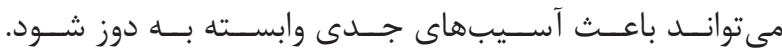

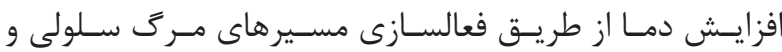

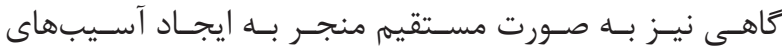

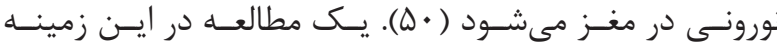

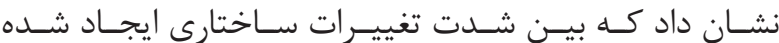

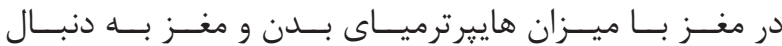

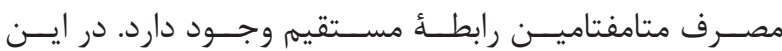

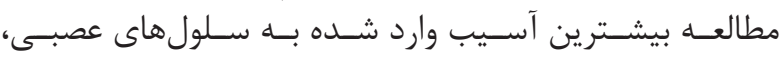

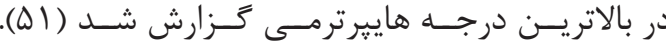

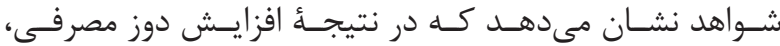

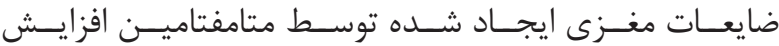

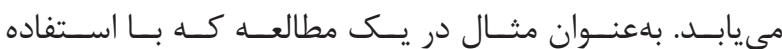

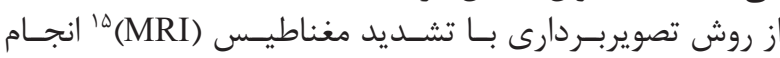

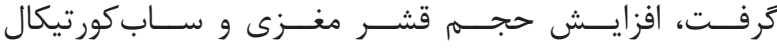

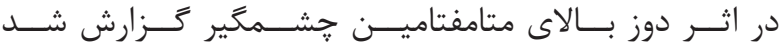

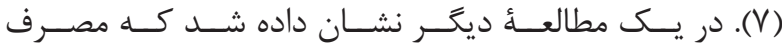

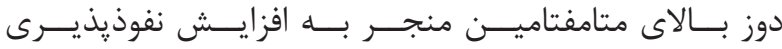

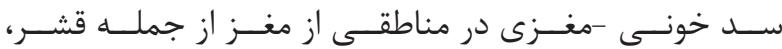

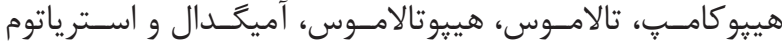

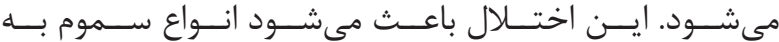

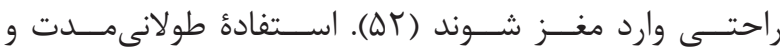

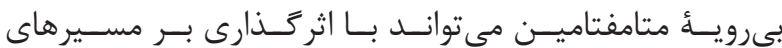

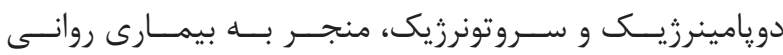

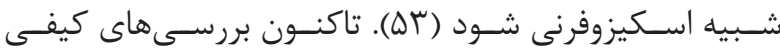

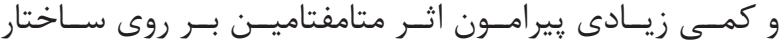

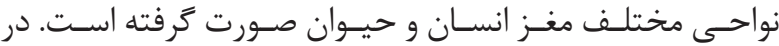

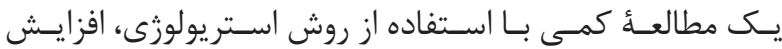

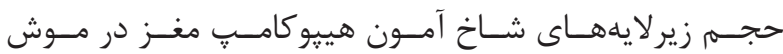

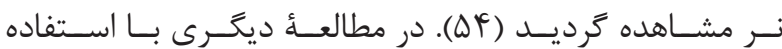

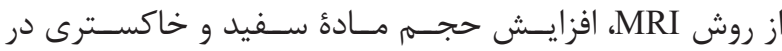

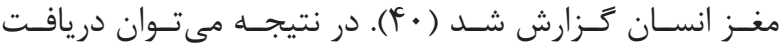

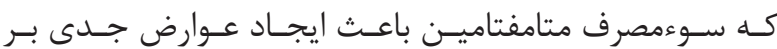

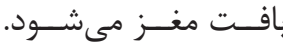

نتيجه

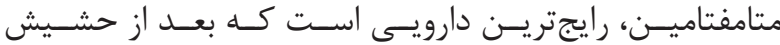

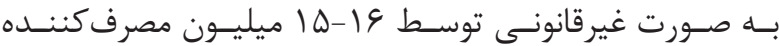

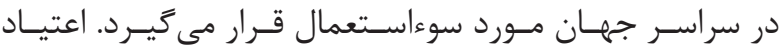

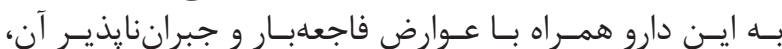

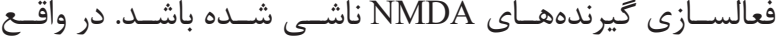

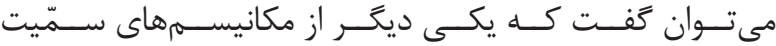

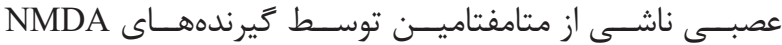

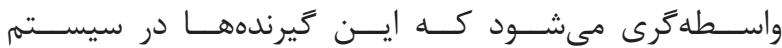

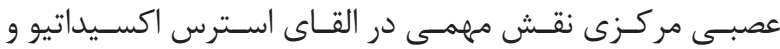

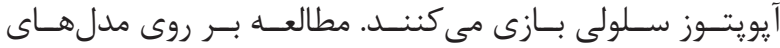

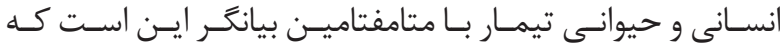

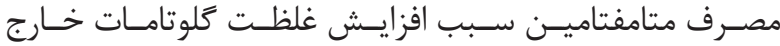

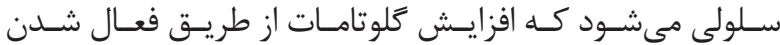

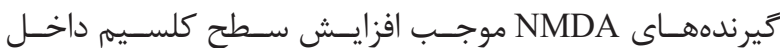

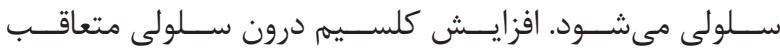

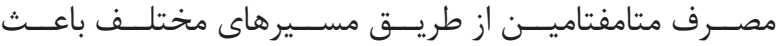

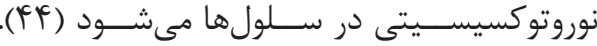

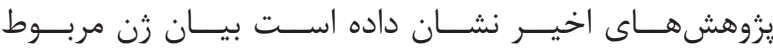

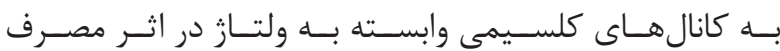

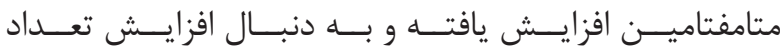

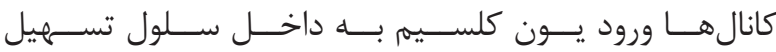

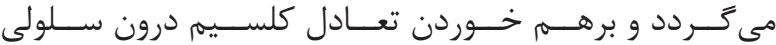

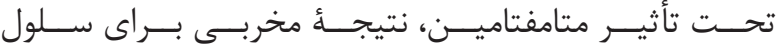

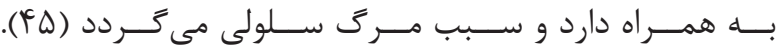

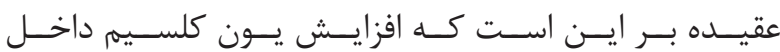

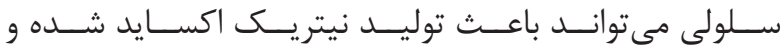

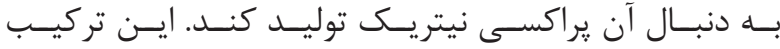

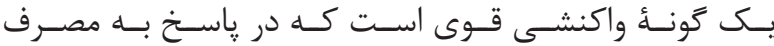

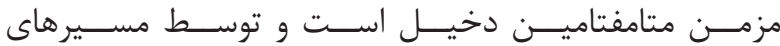

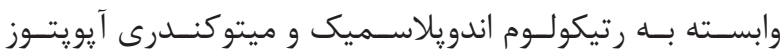

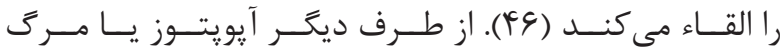

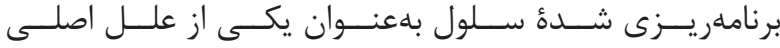

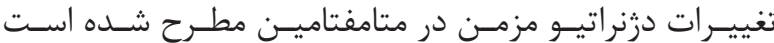

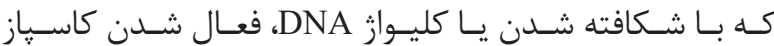

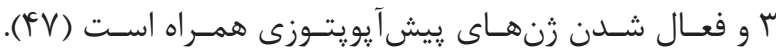

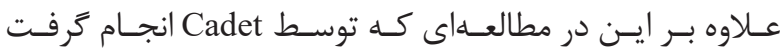

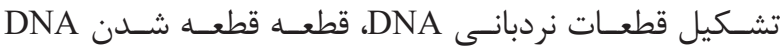

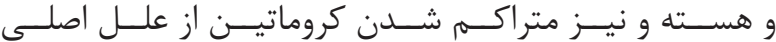

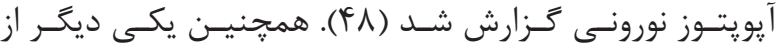

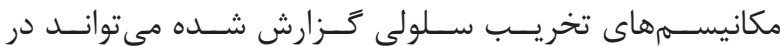

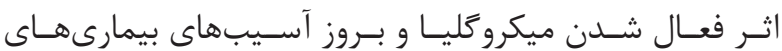

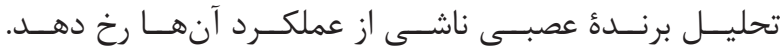

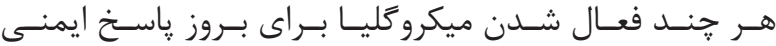

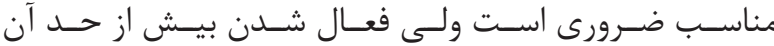

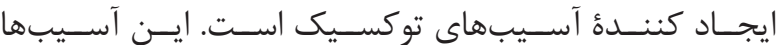

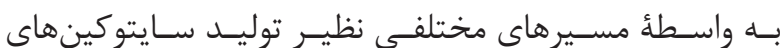

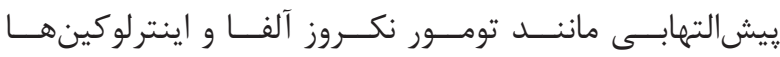

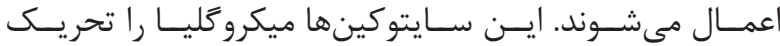

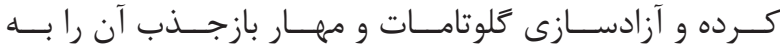

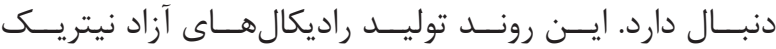

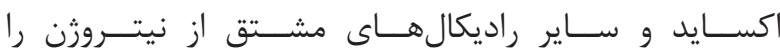




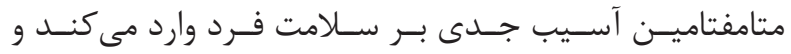

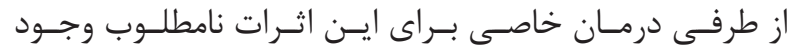

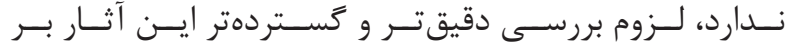

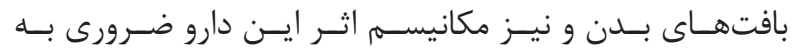

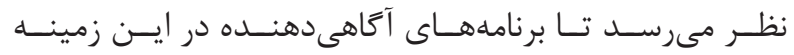

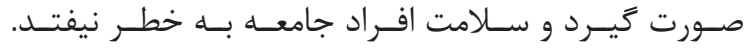

1. Kamali Ardakani R, Nahangi H, Yadegari M, HosseiniSharifabad M. The effects of long-term administration of methamphetamine on the cerebellum of the male mice: a stereological study. Shefaye Khatam. 2014; 2(4): 37-45.

2. Rusyniak DE. Neurologic manifestations of chronic methamphetamine abuse. Psychiatr Clin North Am. $2011 ; 29(3): 641-55$.

3. Won S, Hong RA, Shohet RV, Seto TB, Parikh NI. Methamphetamine-associated cardiomyopathy. Clin Cardiol. 2013; 36(12): 737-42.

4. Allerton M, Blake W. The "Party drug" crystal methamphetamine: risk factor for the acquisition of HIV. Perm J. 2008; 12(1): 56-8.

5. Santos AP, Wilson AK, Hornung CA, Polk Jr HC, Rodriguez JL, Franklin GA. Methamphetamine laboratory explosions: a new and emerging burn injury. J Burn Care Res. 2005; 26(3): 228-32.

6. Alam-Mehrjerdi Z, Mokri A, Dolan K. Methamphetamine use and treatment in Iran: a systematic review from the most populated Persian gulf country. Asian J Psychiatr. 2015; 16: 17-25.

7. Thanos PK, Kim R, Delis F, Ananth M, Chachati G, Rocco MJ, et al. Chronic methamphetamine effects on brain structure and function in rats. PloS One. 2016; 11(6): e0155457.

8. Herman-Stahl MA, Krebs CP, Kroutil LA, Heller DC. Risk and protective factors for nonmedical use of prescription stimulants and methamphetamine among adolescents. J Adolesc Health. 2006; 39(3): 374-80.

9. Karila L, Weinstein A, Aubin HJ, Benyamina A, Reynaud M, Batki SL. Pharmacological approaches to methamphetamine dependence: a focused review. Br J Clin Pharmacol. 2010; 69(6): 578-92.

10. Prakash MD, Tangalakis K, Antonipillai J, Stojanovska L, Nurgali K, Apostolopoulos V. Methamphetamine: effects on the brain, gut and immune system. Pharmacol Res. 2017; 120: 60-7.

11. Marshall JF, O'Dell SJ. Methamphetamine

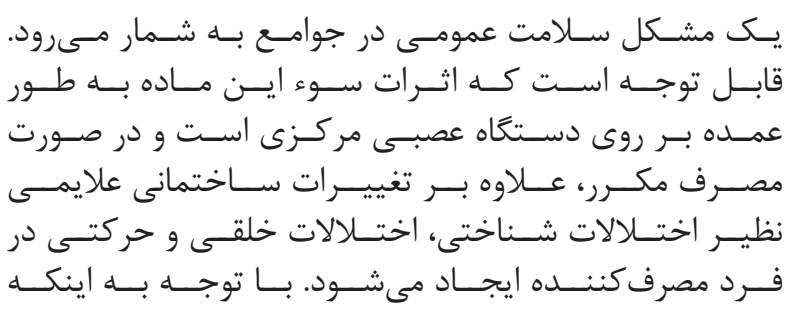

منابع

influences on brain and behavior: unsafe at any speed? Trends Neurosci. 2012; 35(9): 536-45.

12. Killinger B, Shah M, Moszczynska A. Co-adminis tration of betulinic acid and methamphetamine causes toxicity to dopaminergic and serotonergic nerve terminals in the striatum of late adolescent rats. J Neurochem. 2014; 128(5): 764-75.

13. Suzuki O, Hattori H, Asano M, Oya M, Katsumata Y. Inhibition of monoamine oxidase by d-methamphetamine. Biochem Pharmacol. 1980; 29(14): 2071-3.

14. Kitanaka J, Kitanaka N, Takemura M. Modification of monoaminergic activity by MAO inhibitors influences methamphetamine actions. Drug Target Insights. 2006; 1: $19-28$.

15. Ressler KJ, Nemeroff CB. Role of serotonergic and noradrenergic systems in the pathophysiology of depression and anxiety disorders. Depress Anxiety. 2000; 12(1) :2-19.

16. Moszczynska A, Callan SP. Molecular, behavioral, and physiological consequences of methamphetamine neurotoxicity: implications for treatment. J Pharmacol Exp Ther. 2017; 362(3): 474-88.

17. Vallone D, Picetti R, Borrelli E. Structure and function of dopamine receptors. Neurosci Biobehav Rev. 2000; 24(1): 125-32.

18. M Chiu V, O Schenk J. Mechanism of action of methamphetamine within the catecholamine and serotonin areas of the central nervous system. Curr Drug Abuse Rev. 2012; 5(3): 227-42.

19. Panenka WJ, Procyshyn RM, Lecomte T, MacEwan GW, Flynn SW, Honer WG, et al. Methamphetamine use: a comprehensive review of molecular, preclinical and clinical findings. Drug Alcohol Depend. 2013; 129(3): 167-79.

20. Ares-Santos S, Granado N, Moratalla R. The role of dopamine receptors in the neurotoxicity of methamphetamine. J Intern Med. 2013; 273(5): 437-53.

21. Stephans SE, Yamamoto BK. Methamphetamine- 


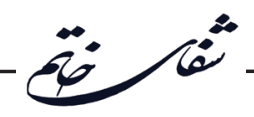

induced neurotoxicity: roles for glutamate and dopamine efflux. Synapse. 1994; 17(3): 203-9.

22. Larsen KE, Fon EA, Hastings TG, Edwards RH, Sulzer D. Methamphetamine-induced degeneration of dopaminergic neurons involves autophagy and upregulation of dopamine synthesis. J Neurosci. 2002; 22(20): 8951-60.

23. Cadet JL, Jayanthi S, Deng X. Speed kills: cellular and molecular bases of methamphetamine-induced nerve terminal degeneration and neuronal apoptosis. Faseb J. 2003; 17(13): 1775-88.

24. Sekine Y, Iyo M, Ouchi Y, Matsunaga T, Tsukada $\mathrm{H}$, Okada $\mathrm{H}$, et al. Methamphetamine-related psychiatric symptoms and reduced brain dopamine transporters studied with PET. Am J Psychiatry. 2001; 158(8): 1206-14.

25. Kish SJ. Pharmacologic mechanisms of crystal meth. Can Med Assoc J. 2008; 178(13): 1679-82.

26. Oláh T, Ocsovszki I, Mándi Y, Pusztai R, Bakay M, Balint E. Opposite effects of serotonin and interferon- $\alpha$ on the membrane potential and function of human natural killer cells. In Vitro Cell Dev Biol-Anim. 2005; 41(5): 165-70.

27. Sekine Y, Ouchi Y, Takei N, Yoshikawa E, Nakamura $\mathrm{K}$, Futatsubashi M, et al. Brain serotonin transporter density and aggression in abstinent methamphetamine abusers. Arch Gen Psychiatry. 2006; 63(1): 90-100.

28. Schmidt CJ, Somalia PK, Hanson GR, Peat MA, Gibb JW. Methamphetamine-induced depression of monoamine synthesis in the rat: development of tolerance. J Neurochem. 1985; 44(3): 852-5.

29. Kishi T, Tsunoka T, Ikeda M, Kitajima T, Kawashima $\mathrm{K}$, Okochi $\mathrm{T}$, et al. Serotonin $1 \mathrm{~A}$ receptor gene is associated with Japanese methamphetamine-induced psychosis patients. Neuropharmacology. 2010; 58(2): 452-6.

30. Kaushal N, R Matsumoto R. Role of sigma receptors in methamphetamine-induced neurotoxicity. Curr Neuropharmacol. 2011; 9(1): 54-7.

31. Hart CL, Marvin CB, Silver R, Smith EE. Is cognitive functioning impaired in methamphetamine users? a critical review. Neuropsychopharmacology. 2012; 37(3): 586-608.

32. Adriani W, Felici A, Sargolini F, Roullet P, Usiello A, Oliverio A, et al. N-methyl-D-aspartate and dopamine receptor involvement in the modulation of locomotor activity and memory processes. Exp Brain Res. 1998; 123(1-2): 52-9.

33. Thanos PK, Kim R, Delis F, Rocco MJ, Cho J, Volkow ND. Effects of chronic methamphetamine on psychomotor and cognitive functions and dopamine signaling in the brain. Behav Brain Res. 2017; 320: 282-90.

34. Simon SL, Domier C, Carnell J, Brethen P, Rawson $\mathrm{R}$, Ling W. Cognitive impairment in individuals currently using methamphetamine. Am J Addict. 2000; 9(3): 222-31.

35. Scott JC, Woods SP, Matt GE, Meyer RA, Heaton $\mathrm{RK}$, Atkinson $\mathrm{JH}$, et al. Neurocognitive effects of methamphetamine: a critical review and meta-analysis. Neuropsychol Rev. 2007; 17(3): 275-97.

36. Murnane KS, Perrine SA, Finton BJ, Galloway MP, Howell LL, Fantegrossi WE. Effects of exposure to amphetamine derivatives on passive avoidance performance and the central levels of monoamines and their metabolites in mice: correlations between behavior and neurochemistry. Psychopharmacology. 2012; 220(3): 495-508.

37. Roussotte FF, Bramen JE, Nunez SC, Quandt LC, Smith L, O'connor MJ, et al. Abnormal brain activation during working memory in children with prenatal exposure to drugs of abuse: the effects of methamphetamine, alcohol, and polydrug exposure. Neuroimage. 2011; 54(4): 3067-75.

38. Henry BL, Minassian A, Perry W. Effect of methamphetamine dependence on everyday functional ability. Addict Behav. 2010; 35(6): 593-8.

39. Paulus MP, Hozack NE, Zauscher BE, Frank L, Brown GG, Braff DL, et al. Behavioral and functional neuroimaging evidence for prefrontal dysfunction in methamphetamine-dependent subjects. Neuropsychopharmacology. 2002; 26(1): 53-63.

40. Thompson PM, Hayashi KM, Simon SL, Geaga JA, Hong MS, Sui Y, et al. Structural abnormalities in the brains of human subjects who use methamphetamine. J Neurosci. 2004; 24(26): 6028-36.

41. Recinto P, Samant ARH, Chavez G, Kim A, Yuan CJ, Soleiman M, et al. Levels of neural progenitors in the hippocampus predict memory impairment and relapse to drug seeking as a function of excessive methamphetamine self-administration. Neuropsychopharmacology. 2012; 37(5): 1275-87.

42. Cadet JL, Krasnova IN. Molecular bases of 
methamphetamine-induced neurodegeneration. Int Rev Neurobiol. 2009; 88: 101-19.

43. Wang S-F, Yen J-C, Yin P-H, Chi C-W, Lee H-C. Involvement of oxidative stress-activated JNK signaling in the methamphetamine-induced cell death of human SH-SY5Y cells. Toxicology. 2008; 246(2-3): 234-41.

44. Kuhn DM, Francescutti-Verbeem DM, Thomas DM. Dopamine quinones activate microglia and induce a neurotoxic gene expression profile. Ann N Y Acad Sci. 2006; 1074(1): 31-41.

45. Andres MA, Cooke IM, Bellinger FP, Berry MJ, Zaporteza MM, Rueli RH, et al. Methamphetamine acutely inhibits voltage-gated calcium channels but chronically up-regulates L-type channels. J Neurochem. 2015; 134(1): 56-65.

46. Tata DA, Yamamoto BK. Interactions between methamphetamine and environmental stress: role of oxidative stress, glutamate and mitochondrial dysfunction. Addiction. 2007; 102(1): 49-60.

47. Cadet JL, Jayanthi S, Deng X. Methamphetamineinduced neuronal apoptosis involves the activation of multiple death pathways. Review Neurotox Res. 2005; 8(3-4): 199-206.

48. Cadet JL, Ordonez SV, Ordonez JV. Methamphetamine induces apoptosis in immortalized neural cells: protection by the proto-oncogene, bcl-2.
Synapse. 1997; 25(2): 176-84.

49. Sekine Y, Ouchi Y, Sugihara G, Takei N, Yoshikawa E, Nakamura K, et al. Methamphetamine causes microglial activation in the brains of human abusers. J Neurosci. 2008; 28(22): 5756-61.

50. Johnson-Davis KL, Fleckenstein AE, Wilkins DG. The role of hyperthermia and metabolism as mechanisms of tolerance to methamphetamine neurotoxicity. Eur $\mathrm{J}$ Pharmacol. 2003; 482(1-3): 151-4.

51. Sharma HS, Kiyatkin EA. Rapid morphological brain abnormalities during acute methamphetamine intoxication in the rat: an experimental study using light and electron microscopy. J Chem Neuroanat. 2009; 37(1): 18-32.

52. Martins T, Baptista S, Gonçalves J, Leal E, Milhazes $\mathrm{N}$, Borges F, et al. Methamphetamine transiently increases the blood-brain barrier permeability in the hippocampus: role of tight junction proteins and matrix metalloproteinase-9. Brain Res. 2011; 1411: 28-40.

53. Hsieh JH, Stein DJ, Howells FM. The neurobiology of methamphetamine induced psychosis. Front Hum Neurosci. 2014; 8: 537. doi: 10.3389/fnhum.2014.00537.

54. Hadizade Asar S, Hosseini-Sharifabad M, Yadegari M. A stereological study on hippocampal subfields following administration of methamphetamine in male mice. IJML. 2016; 3(4): 270-81. 